\title{
Highly Elevated Serum Concentrations of Perfluoroalkyl Substances in Fishery Employees from Tangxun Lake, China
}

\author{
Zhen Zhou, ${ }^{\dagger}$ Yali Shi, ${ }^{\dagger}$ Robin Vestergren, ${ }^{\dagger}$ Thanh Wang, ${ }^{\dagger}$ Yong Liang, ${ }^{*}, \S, \|$ and Yaqi Cai ${ }^{*}{ }^{\dagger}$ \\ ${ }^{\dagger}$ State Key Laboratory of Environmental Chemistry and Ecotoxicology, Research Center for Eco-Environmental Science, Chinese \\ Academy of Sciences, Beijing 100085, China \\ ${ }^{\ddagger}$ Norwegian Institute for Air Research (NILU), 9296 Tromsø, Norway \\ ${ }^{\S}$ School of Medicine, Jianghan University, Hubei Province, Wuhan 430056, China \\ "Key Laboratory of Optoelectronic Chemical Materials and Devices of Ministry of Education, Jianghan University, Hubei Province, \\ Wuhan 430056, China
}

\section{Supporting Information}

ABSTRACT: Increasing production and use of per- and polyfluoroalkyl substances (PFASs) has been reported from China, and a few studies have shown there are subpopulations in China with high and increasing exposure to these chemicals. In this paper, we present a comprehensive exposure assessment of PFASs in fishery employees from Tangxun Lake, China. Exceptionally high serum concentrations of C4 to C12 PFASs were observed in fishery employees $(n=39$, median perfluorooctanesulfonic acid (PFOS) $10400 \mathrm{ng} / \mathrm{mL}$ ) compared to a reference group from the same city $(n=9$, median PFOS $18.7 \mathrm{ng} / \mathrm{mL}$ ). On the basis of the comparison of different exposure pathways, it was concluded that contaminated fish from Tangxun Lake was the primary source of PFAS exposure to
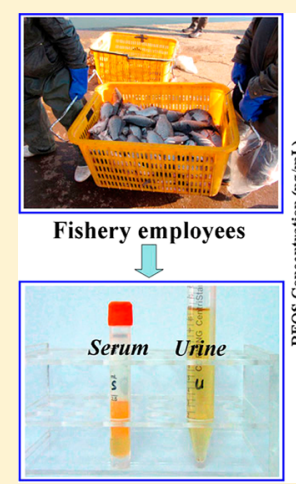
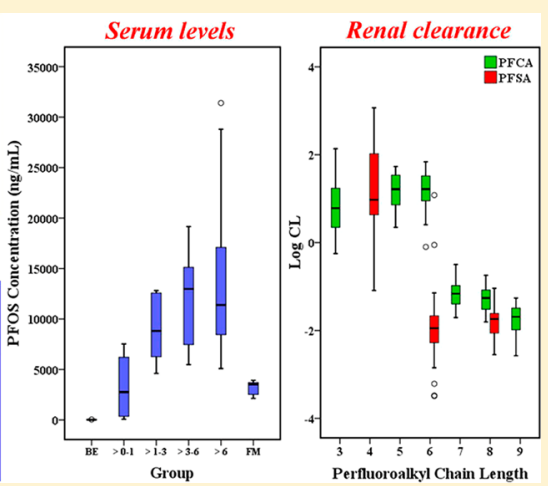
fishery employees, and there was a positive association between serum PFAS concentrations and time of employment in the fishery. PFOS isomer profiles in fishery employees showed a significantly higher proportion of linear PFOS (78.4\%) compared to the background-exposed reference group (66.8\%), reflecting the highly linear PFOS isomer profile $(>90 \%)$ of lake fish. Median renal clearance rates $\left(\mathrm{CL}_{\text {renal }}\right)$ of $\mathrm{C} 4$ to $\mathrm{C} 10$ perfluoroalkyl carboxylic acids (PFCAs) and perfluoroalkanesulfonic acids (PFSAs) ranged from 0.020 to $16.5 \mathrm{~mL} / \mathrm{day} / \mathrm{kg}$ and 0.013 to 9.43 $\mathrm{mL} /$ day/kg, respectively. PFCAs with less than eight perfluoroalkyl carbons were primarily eliminated via urine, whereas other routes of excretion may have contributed to the elimination for long-chain PFCAs and PFSAs. Calculated daily PFOS exposures of fishery employees significantly exceeded tolerable daily intake limits, but clinical blood chemistry parameters were mostly within normal reference ranges. However, additional epidemiological studies are needed to address potential associations between PFAS exposure and health effects in the Tangxun Lake area.

\section{INTRODUCTION}

Per- and polyfluoroalkyl substances (PFASs) are a class of manmade chemicals which display many unique properties such as high thermal and chemical stability and surface activity. ${ }^{1}$ PFASs include a wide range of substances which all contain a perfluoroalkyl moiety of varying carbon chain lengths which is terminated by different functional groups, e.g., carboxylic acid (PFCAs) or sulfonic acid (PFSAs). Since the 1950s, PFASs have been widely used in industrial and commercial applications, including coatings for carpets and fabrics, fastfood contact materials, fire-fighting foams, hydraulic fluids, and pesticides. As a consequence of their long production history and widespread usage, PFASs have been detected in human and wildlife samples from around the world. ${ }^{2,3}$ However, the mechanisms of bioaccumulation and human exposure are only partly understood. ${ }^{4,5}$
Long-chain PFCAs $\left(\mathrm{C}_{n} \mathrm{~F}_{2 n+1} \mathrm{COOH}, n \geq 7\right)$ and PFSAs $\left(\mathrm{C}_{n} \mathrm{~F}_{2 n+1} \mathrm{SO}_{3} \mathrm{H}, n \geq 6\right)$ are of special environmental concern due to their persistence, ${ }^{1}$ bioaccumulation potential, ${ }^{4}$ and toxicology, ${ }^{6}$ and a series of actions have been taken by regulators and the producing industry to reduce the emissions of these compounds. In 2002, perfluorooctanesulfonic acid (PFOS) along with its salts and perfluorooctane sulfonyl fluoride (POSF) was phased out by the major global manufacturer, $^{7}$ and in 2009 the same group of chemicals was listed as new persistent organic pollutants (POPs). ${ }^{8}$ Although PFOS has largely been phased out in Europe, North America,

Received: December 25, 2013

Revised: February 22, 2014

Accepted: March 3, 2014

Published: March 3, 2014 
and Japan, production and use of PFOS and related chemicals have continued after year 2000 in other parts of the world, including China. ${ }^{9}$ The phase-out of long-chain PFASs by the major producers has also led to a wide range of replacement chemicals, which are usually shorter-chain homologues of their predecessors, being introduced on the market. ${ }^{10}$

Human biomonitoring and exposure modeling studies of PFASs have demonstrated that dietary intake is a major exposure pathway for several long-chain PFASs in the general population. ${ }^{5,11}$ However, drinking water intake and ingestion of house dust may also be important exposure pathways in locally contaminated areas $^{12}$ or in subgroups of the population with extensive use of PFAS containing products. ${ }^{13,14}$ The relative importance of dietary intake compared to other exposure pathways may also vary for the different PFAS homologues. ${ }^{15}$ Consumption of fish and shellfish is a major determinant of internal exposure to PFOS and long-chain PFCAs. ${ }^{11}$ For shortchain PFCAs $\left(\mathrm{C}_{n} \mathrm{~F}_{2 n+1} \mathrm{COOH}, n<7\right)$ and PFSAs $\left(\mathrm{C}_{n} \mathrm{~F}_{2 n+1} \mathrm{SO}_{3} \mathrm{H}, n<6\right)$, on the other hand, the lower bioaccumulation potential ${ }^{4}$ indicates that a lower exposure via the diet compared to long-chain homologues is to be expected. ${ }^{15}$ However, little is known about the human exposure, accumulation, and elimination kinetics of shortchain PFAS replacements. ${ }^{16-19}$

The pervasive exposure to PFASs has raised concerns regarding long-term effects in humans. Several epidemiological studies of PFASs in the general population have shown an association between PFOS and PFOA and decreased sperm count, decline of birth weight and size, current thyroid disease, and elevated cholesterol. ${ }^{20-22}$ Meanwhile, it is reported that serum PFOA in occupational exposed people was associated with total cholesterol ( $\mathrm{CHOL})$, total bilirubin, serum aspartate transaminase (AST), and thyroid parameters. ${ }^{23,24}$ However, the findings of statistically significant associations between PFAS exposure and clinical biomarkers vary between different studies, ${ }^{25,26}$ and so far the evidence for human health effects as a consequence of PFAS exposure remain largely inconclusive.

The overarching aim of this study was to characterize human exposure to PFASs in fishery employees and their families around Tangxun Lake, China. In our previous study, we observed highly elevated concentrations of a wide range of PFASs in the water of Tangxun Lake, ${ }^{27}$ but human exposure has not been investigated in this area so far. The specific objectives of this study were to (i) measure concentrations of PFCAs, PFSAs, and perfluorooctane sulfonamide (FOSA) in lake fish, indoor dust, drinking water, human serum, and urine samples; (ii) investigate associations between internal exposure to PFASs and duration of exposure; (iii) quantify renal clearance rates for long- and short-chain PFASs; and (iv) investigate statistical correlations between PFAS serum concentrations and biochemical parameters, including blood lipids, hepatic clinical parameters, kidney clinical parameters, serum albumin, blood glucose, and thyroid hormones.

\section{MATERIALS AND METHODS}

Study Area and Sampling Design. Tangxun Lake is a shallow lake with a current surface area of approximately 32 $\mathrm{km}^{2}$. It is located in Hubei Province, which is one of the production bases of several small-scale fluorochemical manufacturers and users in China. ${ }^{9}$ In our previous study, high concentrations of PFASs, including short-chain PFASs, were observed in water samples from Tangxun Lake. The average concentrations of PFBS and PFBA were $3660 \pm 558$ $\mathrm{ng} / \mathrm{L}$ and $4770 \pm 1400 \mathrm{ng} / \mathrm{L}$, respectively. ${ }^{27}$ Several fisheries which supply freshwater fish to the general population in the area are located around Tangxun Lake, and fish is a particularly important part of the diet for fishery employees.

Blood and urine samples were collected from fishery employees $(n=39$; male $=38$; female $=1)$, their family members $(n=7$; male $=1$; female $=6)$, and backgroundexposed reference persons $(n=9$; male $=5$; female $=4)$ who lived in the same region but more than $30 \mathrm{~km}$ away from Tangxun Lake. Generally, the fishery employees lived in the surrounding of the lake and had meals in the fishery cafeteria during working days, and the fish served in the cafeteria was primarily from Tangxun Lake. Fishery employees habitually brought fish from Tangxun Lake to their home, so the family members of fishery employees also obtained a part of their fish consumption from Tangxun Lake.

Whole blood samples were collected in plain tubes, centrifuged at $3000 \mathrm{~g}$ for $10 \mathrm{~min}$, and separated for serum. Morning urine samples were collected in $50 \mathrm{~mL}$ PP tubes. Serum and urine samples were stored in PP tubes at $-20{ }^{\circ} \mathrm{C}$ until further analysis. On the date of blood and urine sampling, a questionnaire for self-completion was sent to the study participants to obtain information such as age, gender, body height, body weight, smoking habits, drinking habits, exposure to environmental tobacco smoke, and environmental or occupational exposures to PFASs. Meanwhile, trained interviewers administered a standardized questionnaire about the time of employment in the fishery, fish consumption, drinking water consumption, and species of fish serving in the fishery cafeteria. Since fishery employees did not prepare the food portions themselves it was difficult for the study participants to provide accurate dietary intake which would allow examination of individual differences in dietary intake. However, based on the questionnaires, we calculated an average value of $100 \mathrm{~g} /$ day for fish consumption of fishery employees based on the total consumption per week. According to the questionnaires, various fish species and mallard were served in the fishery cafeteria. The demographic information for participants is shown in Table S3.

Drinking water $(n=2)$ and indoor dust samples $(n=3)$ were collected from the residence of fishery employees. Aquatic biota samples $(n=60)$ were collected from the fishery in Tangxun Lake. Indoor dust samples were collected from one office and two large staff rooms, and drinking water samples were collected from the water faucets around the residence of fishery employees. Although the number of indoor dust and drinking water samples is low, the samples would be roughly representative of the exposure situation of fishery employees. Further information about these samples is detailed in the Supporting Information. The sampling and questionnaire were approved by the ethical commission of the Research Center for Eco-Environmental Science, Chinese Academy of Sciences, and it was conducted in accordance with the ethical principles for medical research involving human subjects in China. Informed consent was obtained from all study participants prior to the study.

Chemicals. A total of 14 PFASs were analyzed, including perfluorobutanoic acid (PFBA), perfluoropentanoic acid (PFPeA), perfluorohexanoic acid (PFHxA), perfluoroheptanoic acid (PFHpA), perfluorooctanoic acid (PFOA), perfluorononanoic acid (PFNA), perfluorodecanoic acid (PFDA), perfluoroundecanoic acid (PFUnDA), perfluorododecanoic acid 


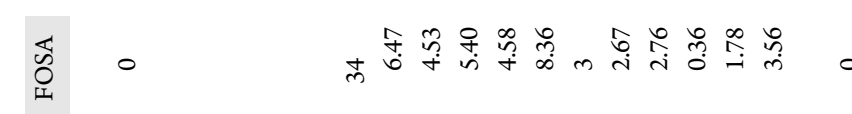

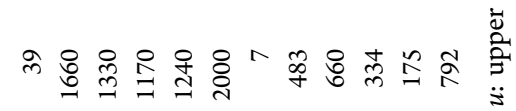

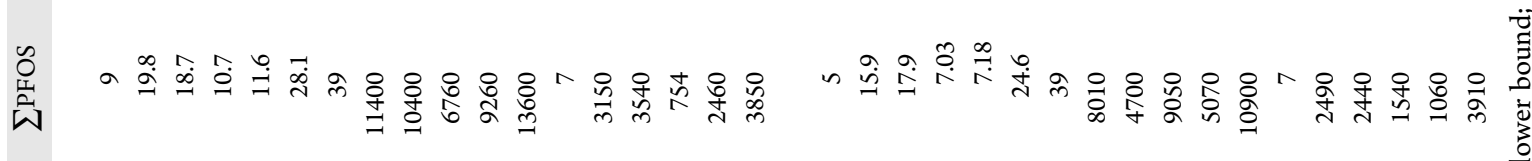

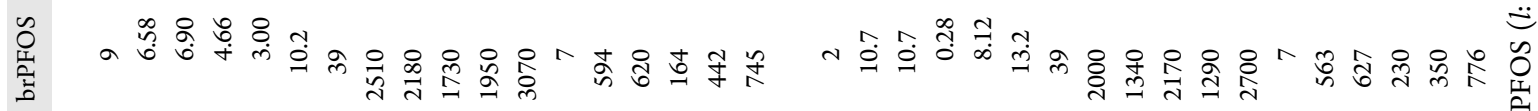

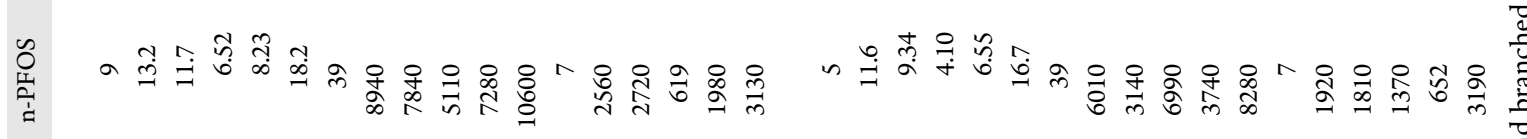

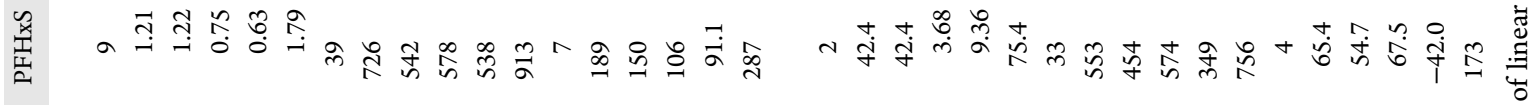

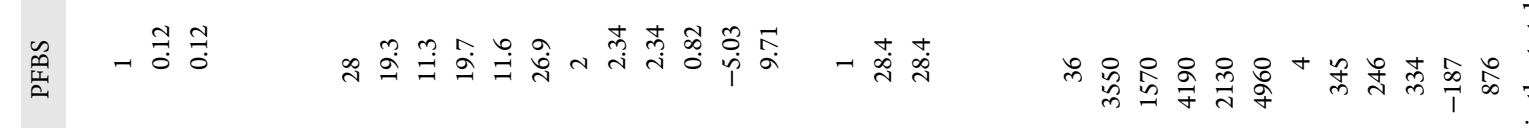

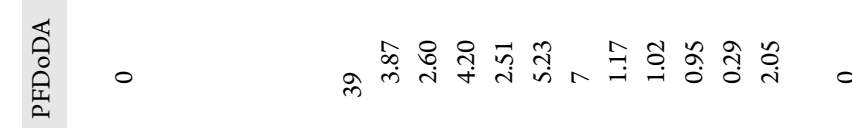

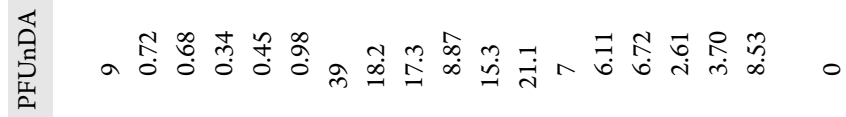

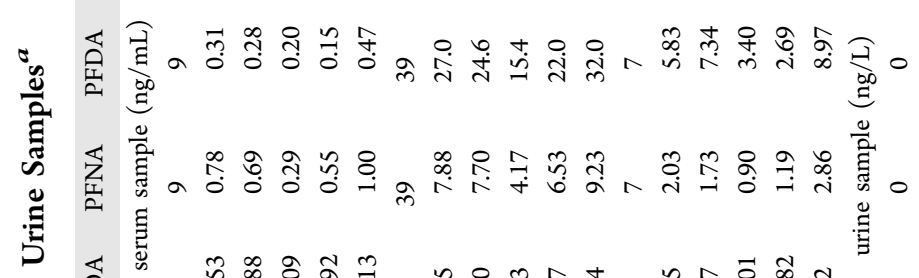

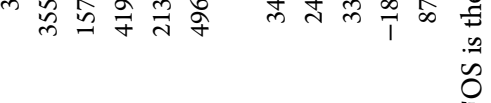

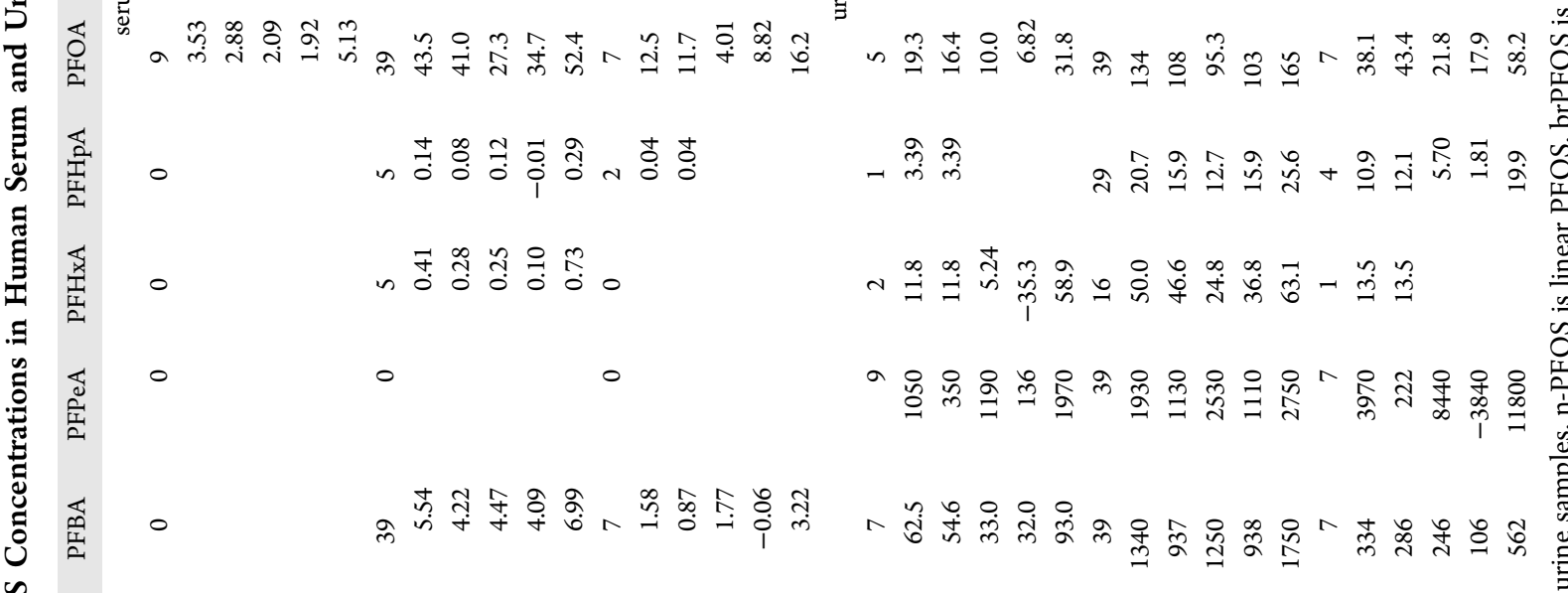

t:

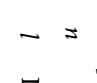

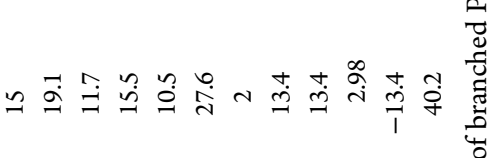

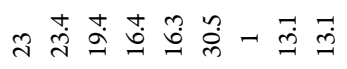

立

(⿹) 
(PFDoDA), perfluorotridecanoic acid (PFTrDA), perfluorobutanesulfonate (PFBS), perfluorohexanesulfonate (PFHxS), PFOS, and FOSA. Nonlabeled calibration standards and isotopically labeled internal standards were obtained from Wellington Laboratories (Ontario, Canada). A list of the standards used is shown in Table $\mathrm{S} 1$ of the Supporting Information.

Extraction and Analysis of PFASs. Serum samples were prepared according to a procedure adapted from Yeung et al., ${ }^{28}$ using acetonitrile extraction and cleanup by OASIS WAX-SPE cartridges. For urine samples, we employed a previously reported extraction method with minor modifications. ${ }^{14}$ The details of sample extraction are shown in the Supporting Information.

PFAS analysis was accomplished by high performance liquid chromatography (Ultimate 3000 HPLC, Dionex, Sunnyvale, CA, USA) equipped with an electrospray ionization tandem mass spectrometer (API 3200, Applied Biosystems/MDS SCIEX, USA) operated in negative mode. A detailed description of analyte separation and instrument parameters is provided in the Supporting Information.

Quality Assurance and Quality Control (QA/QC). Procedural blanks were conducted for each batch of 10 samples to check for possible laboratory contamination and interferences (Table S2). Quantification was performed using the internal standard method (isotopic dilution) for all target analytes. Branched isomers of PFOS were identified as one or several peaks eluting before the peak of linear PFOS. Quantification of the sum of branched PFOS (brPFOS) isomers was performed using the isotope-labeled internal standard for linear PFOS (n-PFOS), but the average concentrations of the two transitions for PFOS $(\mathrm{m} / \mathrm{z} 498.9-$ 79.9 and 498.9-99.0) were used. The method limit of quantification (MLQ) was considered to be the lowest concentration resulting in a signal-to-noise ratio $(\mathrm{S} / \mathrm{N}) \geq 10$ for each compound. When PFAS was observed in the procedural blanks, MLQ for the PFAS was determined as 10 times the standard deviation of the procedural blank concentrations $(n=7)$. The mean matrix spike recovery $(n=$ 5) of PFASs ranged from 85 to $117 \%, 89$ to $117 \%, 75$ to $109 \%$, 81 to $103 \%$, and 88 to $107 \%$ for water, indoor dust, aquatic biota, serum, and urine samples, respectively (Table S2). Duplicate samples and calibration check standards showed excellent reproducibility with a mean standard deviation of $\sim 10 \%$ for the PFASs. Further information about quality assurance and quality control is detailed in the Supporting Information.

Blood Clinical Chemistry Detection. Biochemical parameters, including blood lipids [total cholesterol (CHOL), triglycerides (TG), high-density lipoprotein cholesterol (HDLchol), and low-density lipoprotein cholesterol (LDL-chol)], hepatic clinical parameters [alanine transaminase (ALT), aspartate transaminase (AST), alkaline phosphatase (ALP)], kidney clinical parameters [blood urea nitrogen (BUN), creatinine (CRE), uric acid (UA)], serum albumin (ALB), blood glucose (GLU), and thyroid hormones [free triiodothyronine (FT3) and free thyroxine (FT4)], were analyzed for all blood samples. All the above clinical assays were done by clinical professionals with experience in their calibration. The details of the blood clinical chemistry are shown in the Supporting Information.

Variable Calculation and Statistical Analysis. Daily renal clearance $\left(\mathrm{CL}_{\text {renal, }} \mathrm{mL} / \mathrm{day} / \mathrm{kg}\right)$ of PFASs was calculated based on the paired serum and urinary concentrations with the following equation: ${ }^{29}$

$$
\mathrm{CL}_{\text {renal }}=C_{\text {urine }} \times V /\left(C_{\text {serum }} \times W\right)
$$

where $\mathrm{C}_{\text {urine }}$ is the concentration of PFAS in urine $(\mathrm{ng} / \mathrm{L}), V$ is daily urine excretion volume (L/day, $1.2 \mathrm{~L}$ for females and 1.4 $\mathrm{L}$ for males), ${ }^{30} C_{\text {serum }}$ is the concentration of PFAS in serum $(\mathrm{ng} / \mathrm{mL})$, and $W$ is body weight $(\mathrm{kg})$.

Daily intake of PFASs for fishery employees in Tangxun Lake through drinking water (WI, $\mathrm{ng} / \mathrm{kg}_{\mathrm{bw}} /$ day), indoor dust (DI, $\mathrm{ng} / \mathrm{kg}_{\mathrm{bw}} /$ day), and fish consumption (FI, $\mathrm{ng} / \mathrm{kg}_{\mathrm{bw}} /$ day) was calculated using the following equations modified from Zhang et al., Björklund et al., Bao et al., and Holzer et al. ${ }^{31-34}$

$$
\begin{aligned}
& \mathrm{WI}=C_{\mathrm{w}} \times V_{\mathrm{w}} / W \\
& \mathrm{DI}=C_{\mathrm{d}} \times W_{\mathrm{d}} / W \\
& \mathrm{FI}=C_{\mathrm{f}} \times M_{\mathrm{f}} / W
\end{aligned}
$$

where $C_{\mathrm{w}}$ is the concentration of PFAS in drinking water (ng/ $\mathrm{L}), V_{\mathrm{w}}$ is the daily consumption of drinking water $(2 \mathrm{~L} /$ day $), W$ is body weight (average: $69.9 \mathrm{~kg}_{\mathrm{bw}}$ ), $C_{\mathrm{d}}$ is the concentration of PFAS in indoor dust $(\mathrm{ng} / \mathrm{g}), W_{\mathrm{d}}$ is daily weight of dust $(0.06 \mathrm{~g} /$ day), $C_{\text {fish }}$ is the average PFAS concentrations of fish and mallard muscle in Tangxun Lake (ng/g ww), and $M_{\mathrm{f}}$ is the daily consumption of fish $(100 \mathrm{~g} /$ day $)$.

Statistical analyses, including spearman rank correlation analysis, linear regression, one-way analysis of variance (ANOVA), and multiple regression, were performed using IBM PASW Statistics 18.0 (SPSS Inc., 1993-2007). Smoking and drinking status were not included in the final analyses because they were not significantly associated with any clinical chemistry outcome. Age and body mass index (BMI, weight in kilograms divided by the square of measured height in meters) were not associated with the levels of PFOS, PFHxS, and PFOA but were associated with some clinical chemistry outcomes, such as TG, HDL-chol, LDL-chol, ALT, and BUN. Therefore, the outcomes were adjusted for the covariant (age and BMI). Detailed statistical analyses are shown in the Supporting Information.

\section{RESULTS AND DISCUSSION}

PFAS Concentrations in Human Serum. A summary of the quantified concentrations of PFASs in human serum is given in Table 1 . Among the 14 target analytes, PFBA, PFOA, PFNA, PFDA, PFUnDA, PFDoDA, PFHxS, and PFOS were detected in all serum samples of fishery employees and fishery family members. PFBS was detected in $72 \%$ and $28 \%$ of the fishery employees and fishery families, respectively, and PFHpA and PFHxA were only infrequently detected. Long-chain PFSAs and PFCAs, except PFDoDA, were detected in all samples in the background-exposed reference group, but short-chain PFCAs were not observed in the background-exposed reference group, and PFBS was detected only in one serum sample at a concentration of $0.12 \mathrm{ng} / \mathrm{mL}$.

The median serum concentrations of PFOS and PFOA in the background-exposed reference group were $18.7 \mathrm{ng} / \mathrm{mL}$ (8.67$43.4 \mathrm{ng} / \mathrm{mL})$ and $2.88 \mathrm{ng} / \mathrm{mL}(1.88-8.86 \mathrm{ng} / \mathrm{mL})$, respectively. In fishery employees and their family members, the serum concentrations of all PFASs were significantly higher (1-3 orders of magnitude) than those in the backgroundexposed reference group. PFOS was the dominant PFAS in the serum of fishery employees and their family members, followed 

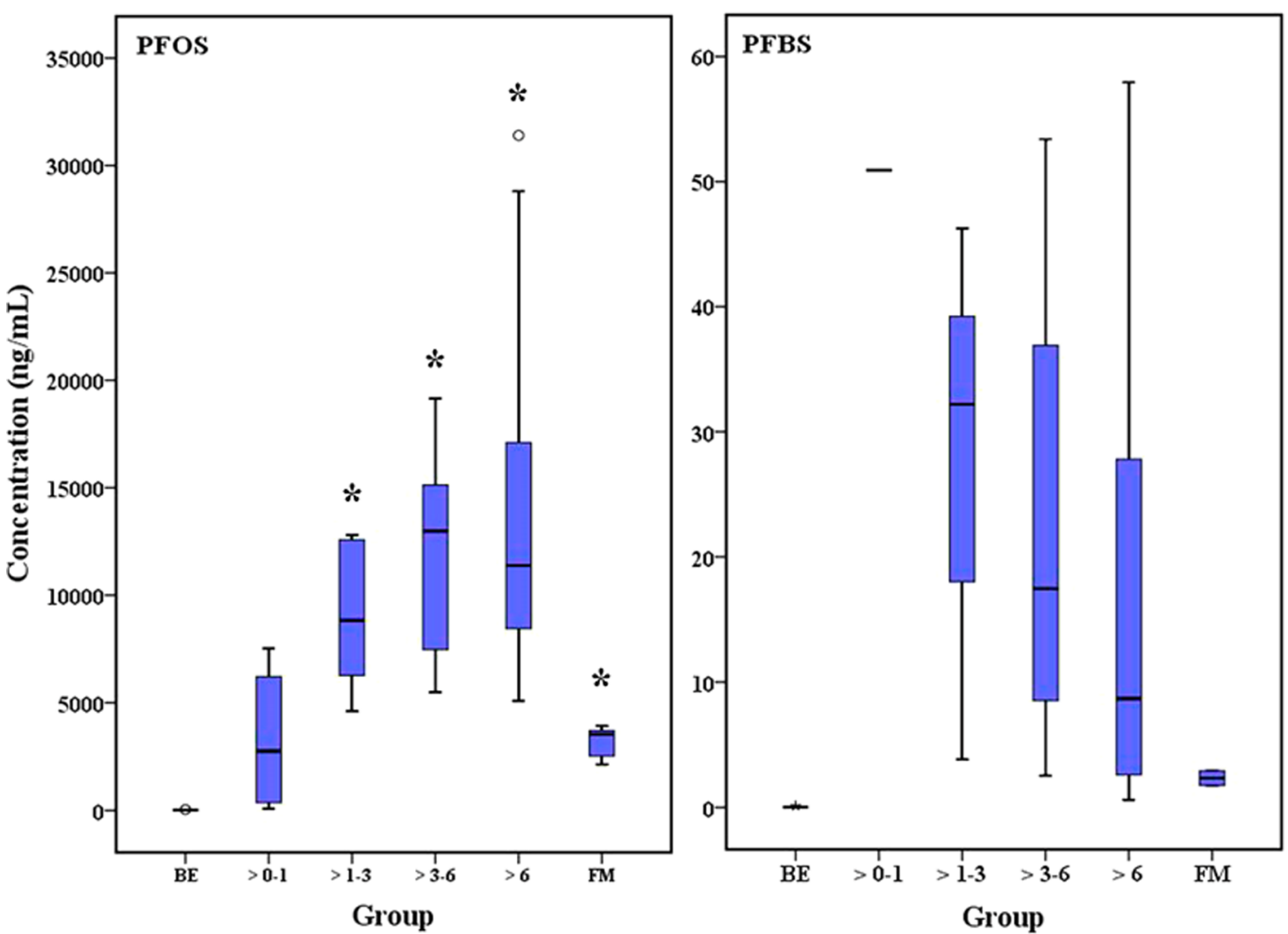

Figure 1. PFOS and PFBS concentrations in serum samples. According to the years of work in the fishery, employees were divided into four groups: $>0-1$ year, > 1-3 years, > 3-6 years, and > 6 years; BE: background-exposed reference persons; FM: family members of fishery employees. (The lower and upper limits of the whiskers indicate $5 \%$ and $95 \%$ values, respectively; boxes extend from 25 th to 75 th percentiles; horizontal lines within the boxes represent medians; and circles below or above the whiskers indicate outlier values; $* p<0.05$, significantly different from $\mathrm{BE}$ ).

by PFHxS, accounting for an average of $93 \%$ and $5.9 \%$ (fishery employees) and $93 \%$ and $5.6 \%$ (family members) of total PFASs, respectively. The median serum concentrations of PFOS and PFHxS in fishery employees were $10400 \mathrm{ng} / \mathrm{mL}$ $(82.6-31400 \mathrm{ng} / \mathrm{mL})$ and $542 \mathrm{ng} / \mathrm{mL}(0.77-2470 \mathrm{ng} / \mathrm{mL})$, respectively, while those in family members of fishery employees were $3540 \mathrm{ng} / \mathrm{mL}(2140-3920 \mathrm{ng} / \mathrm{mL})$ and 150 $\mathrm{ng} / \mathrm{mL}(88.2-406 \mathrm{ng} / \mathrm{mL})$, respectively.

The serum concentrations of PFOS and PFHxS in fishery employees and their family members were $2-3$ orders of magnitude higher compared to all previous biomonitoring studies in the general population in China ${ }^{35}$ and other parts of the world. ${ }^{3,5}$ The arithmetic mean concentrations of PFOS in fishery employees $(11400 \pm 6760 \mathrm{ng} / \mathrm{mL})$ were even more than 1 order of magnitude higher than those in retired fluorochemical production workers from $3 \mathrm{M}$ Company. ${ }^{36}$ While not a traditional "occupational" exposure in the sense that the fishery employees are being exposed as a result of direct contact with or inhalation of PFASs, ${ }^{37,38}$ certainly their elevated exposures is closely linked to their work in a fishery. The elevated concentrations of several long-chain PFCAs are generally in line with previous studies of fishermen from contaminated areas. ${ }^{34}$ Interestingly, PFBA and PFBS, which are not considered to be bioaccumulative, ${ }^{4}$ were observed at comparable concentrations as the long-chain PFCAs. The elevated concentrations of short-chain PFASs in fishery employees indicate a high recent exposure and highlights the need to better understand the exposure pathways and elimination kinetics of these compounds (see following sections).

Aquatic Biota. Ten out of 14 PFASs were quantified in all aquatic biota samples (Figure S1), including PFBA, PFOA, PFNA, PFDA, PFUnDA, PFDoDA, PFBS, PFHxS, PFOS, and FOSA. Consistent with our previous study, ${ }^{27}$ short-chain PFCAs, except for PFBA, were only infrequently detected in aquatic biota samples. PFOS was the dominant PFAS in aquatic biota samples, accounting for 63\% (in shrimp) to $94 \%$ (in mallard) of the total PFASs. The highest PFOS concentrations were found in mallard muscle, ranging from 574 to $818 \mathrm{ng} / \mathrm{g}$ ww. The concentrations of PFOS in fish muscle were generally higher (170-1140 ng/g ww) than those in soft tissues of river snails (144-216 ng/g ww) and whole-body homogenates of shrimp (165-287 ng/g ww; Table S4). Previous studies have reported significantly higher PFOS concentrations in piscivorous fish than nonpiscivorous fish. ${ }^{34,39}$ In our study, higher PFOS and PFHxS concentrations were observed in omnivore fish (common carp and yellow catfish) compared to herbivore fish (grass carp and white amur breans). Slightly lower PFOS and PFHxS concentrations were found in aquatic animals feeding on plankton, algae, organic impurities, and detritus (bighead carp, silver carp, shrimp, and river snails). Unfortunatly, no piscivore fish were collected and analyzed from Tangxun Lake, which limits the discussion of differences in PFAS concentration between fish species with different feeding habits. Nevertheless, it is interesting that FOSA displayed an opposite trend compared to PFOS with the lowest concentrations in mallard muscle (<MLQ-2.74 ng/g 
ww) and highest concentrations in soft tissues of river snails (46.4-64.6 ng/g ww) and whole-body homogenates of shrimp (92.7-110 ng/g ww; Table S4). A statistically significant positive association between PFOS and FOSA was also observed in silver carp $\left(p<0.01, r^{2}=0.89\right)$ and bighead carp $\left(p<0.01, r^{2}=0.73\right)$ in Tangxun Lake, which is in line with previous findings in forage fish species from Lake Ontario. ${ }^{40,41}$ The relatively high FOSA to PFOS ratios in shrimp and river snail (Table S5, mean ratio: 0.430 and 0.287 , respectively) may indicate that aquatic species feeding on the benthos receive a higher exposure to PFOS precursors which are strongly hydrophobic and primarily partition to the sediments. ${ }^{42,43} \mathrm{~A}$ higher capability in fish species to metabolize PFOS precursors, ${ }^{44}$ on the other hand, may explain the shift in FOSA to PFOS ratios at a higher trophic position. Overall, the trends in PFOS and FOSA concentrations between different aquatic species indicate that uptake and metabolism of PFOS precursors ${ }^{42}$ may play an important role in the accumulation of PFOS in the Tangxun Lake food web.

Exposure Pathways of PFASs of Fishery Employees. On the basis of our previous study, ${ }^{27}$ it was hypothesized that high consumers of fish from Tangxun Lake would have elevated concentrations of PFASs in their serum. In order to investigate if other exposure media could make a significant contribution to the total exposure of fishery employees, indoor dust and drinking water samples were also analyzed. In contrast to the PFAS concentrations in aquatic food samples, which were among the highest reported globally, the PFAS concentrations in indoor dust and drinking water samples (Table S6) were similar to those reported from other parts of China ${ }^{31,45}$ and other countries. ${ }^{32}$ On the basis of the deterministic exposure calculations described in eqs $1-3$, we estimated the intake of a range of PFASs via drinking water, ingestion of house dust, and fish consumption (Table S7). Intake of individual PFASs via drinking water and dust ingestion were generally in the same range $\left(<0.01\right.$ to $0.19 \mathrm{ng} / \mathrm{kg}_{\mathrm{bww}} /$ day $)$ and comparable to other studies from Europe and North America. ${ }^{46}$ In contrast, the estimated daily intake of PFASs through fish consumption were many orders of magnitude higher than those determined for background exposed populations ${ }^{15}$ ranging from 0.92 to 621 $\mathrm{ng} / \mathrm{kg}_{\mathrm{bw}} /$ day for individual PFAS homologues. The comparison of different exposure pathways demonstrates that fish consumption is a primary source of exposure to PFASs for fishery employees in Tangxun Lake. However, refined food consumption data for probabilistic intake calculations together with toxicokinetic modeling tools would be needed to evaluate the consistency between external exposure estimates and measured PFAS serum concentrations.

To further elucidate how the duration of exposure affects the serum concentrations of PFASs in fishery employees, the measurements of PFASs in serum were grouped on the basis of the time of employment in the fishery into four groups $(>0-1$ year, $n=4$; > $1-3$ years, $n=6$; $>3-6$ years, $n=5$; and $>6$ years, $n=24$; Figure 1 ). The arithmetic mean serum PFOS concentrations in fishery employees increased with the increasing years worked at the fishery from $3280(>0-1$ year) to $8980(>1-3$ years) to $12000(>3-6$ years $)$ to 13300 $\mathrm{ng} / \mathrm{mL}$ ( $>6$ years). PFOS serum concentrations in family members of fishery employees (FM) were comparable to employees who had worked for less than one year in the fishery but significantly higher than those of the background-exposed reference population (BE; $p<0.001$ ). The trend of increasing serum concentrations from the background-exposed reference group to family members to fishery employees for all PFASs (Figure S2) provides further confidence in the conclusion that consumption of fish from Tangxun Lake was the primary exposure pathway leading to significantly elevated exposure. However, different trends with respect to the time of employment were observed for different PFASs. While PFOS, PFHxS, PFDA, and PFUnDA displayed a trend of increasing concentrations with increasing time of employment at the fishery (Figure S2), PFBS and PFBA (Figure S2) displayed decreasing or constant serum concentrations with increasing time of employment at the fishery. According to first order elimination kinetics it would take four half-lives to reach $93.75 \%$ of steady-state serum concentrations. On the basis of a geometric mean elimination half-lives of 4.8 and 7.3 years, it would take approximately 20 and 30 years of chronic exposure to reach a pseudo steady-state situation for PFOS and PFHxS, respectively. ${ }^{36}$ For PFBS, on the other hand, a pseudo steadystate situation (corresponding to four half-lives) would be reached in approximately 100 days. ${ }^{47}$ The different trends of PFAS serum concentrations with time of employment may, therefore, to a large extent reflect the differences in elimination rates between long- and short-chain PFASs. However, it should be noted that PFOA, PFNA, and PFDoDA, which also have elimination half-lives on the order of several years, ${ }^{29,36}$ did not display consistently increasing serum concentrations with time of employment (Figure S2). Thus, additional factors related to the exposure and/or toxicokinetics of PFASs may be important to explain the concentrations of PFASs in fishery employees. For instance, it is reasonable to assume that the concentration of PFASs in Tangxun Lake and resulting exposure via fish consumption has increased over the past 10 years as a consequence of increasing POSF production. ${ }^{9}$ Meanwhile, the diverging trends between, e.g., PFNA and PFOS might also be indicative of different mechanisms leading to accumulation of PFASs in humans for PFCAs vs PFSAs. Further studies including the temporal aspects of dietary exposure and improved data regarding dietary intake, therefore, help to better understand the exposure to PFASs in fishery employees from Tangxun Lake.

On the basis of the comparison of different exposure pathways, we previously concluded that contaminated fish from Tangxun Lake was the primary source of PFAS exposure to fishery employees and their families. However, as fish samples from Tangxun Lake contained considerable amounts FOSA (Figure S1), the calculated intake of PFOS via fish consumption may represent an underestimation of internal exposure to PFOS since it neglects the uptake and metabolism of PFOS precursors. ${ }^{42}$ The elevated serum concentrations of FOSA in fishery employees compared to the reference group (Figure S2) further demonstrates that indirect exposure to PFOS precursors may account for a significant part of the internal exposure to PFOS. In order to evaluate the relative importance of precursor exposure to the internal exposure of PFOS in fishery employees, isomer profiles in different exposure media (Figure 2A), serum samples (Figure 2B), and urine samples (Figure 2C) were investigated. On the basis of the assumptions that (i) PFOS produced by electrochemical fluorination yields a relatively consistent pattern of $70 \%$ linear and $30 \%$ branched PFOS isomers, ${ }^{10}$ (ii) branched PFOS precursors are metabolized more efficiently, ${ }^{48,49}$ and (iii) branched PFOS isomers are excreted more rapidly via urine, ${ }^{29,50}$ it has previously been hypothesized that enrichment of branched PFOS isomers (n-PFOS $/ \Sigma$ PFOS < 70\%) in 


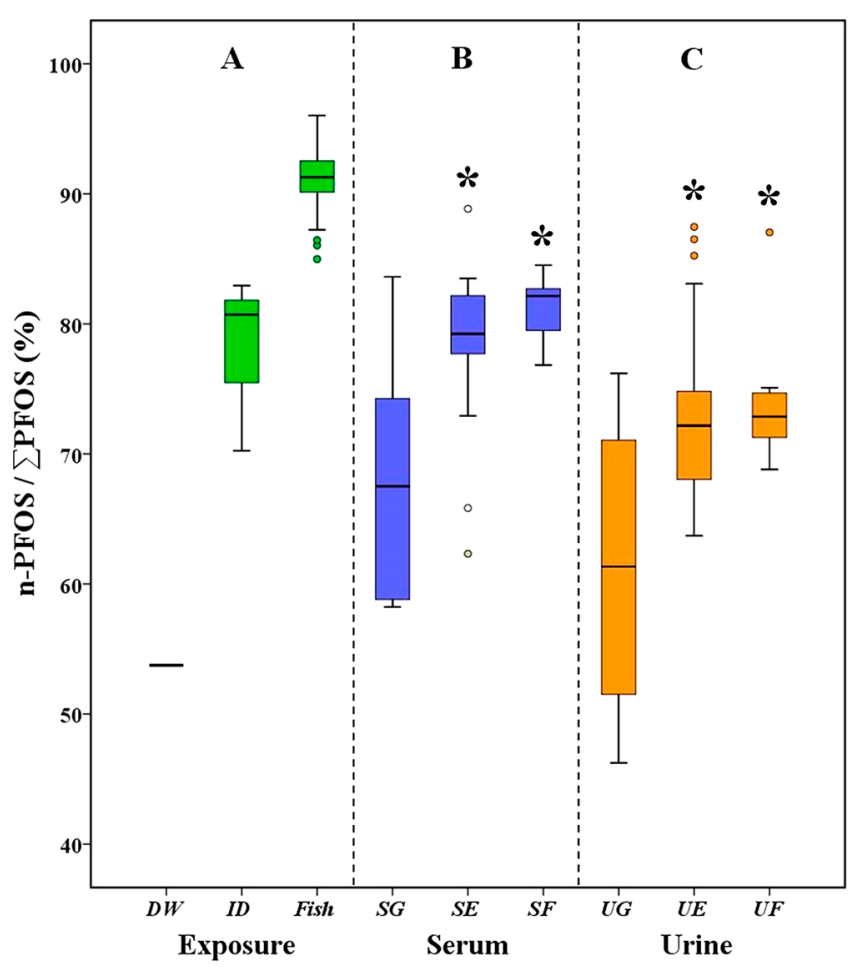

Figure 2. The ratio of $\mathrm{n}$-PFOS concentration to $\sum$ PFOS concentration in different exposure media (A), human serum (B), and human urine (C) samples, including drinking water (DW), indoor dust (ID), fish (Fish), human serum (SG, background-exposed reference persons; SE, fishery employee; SF, fishery family), and human urine (UG, background-exposed reference persons; UE, fishery employee; UF, fishery family). (The lower and upper limits of the whiskers indicate $5 \%$ and $95 \%$ values, respectively; boxes extend from 25th to 75th percentiles; horizontal lines within the boxes represent medians; circles below or above the whiskers indicate outlier values; * $p<0.05$, significantly different from SG or UG.)

human serum can be used as a biomarker of precursor exposure. ${ }^{42}$ Serum samples of fishery employees and family members displayed similar isomer profiles with a 79.2 and 82.1 median percentage of linear PFOS (Figure 2B), whereas a statistically different isomer profile (fishery employee, $p<$ 0.001; family member, $p=0.007$ ) was observed in the background-exposed reference population (median n-PFOS/ $\Sigma$ PFOS of $67.5 \%$ ). The relative enrichment of linear PFOS in fishery employees compared to the reference group and other studies ${ }^{42}$ can to a large extent be explained by the highly linear PFOS isomer in fish samples from Tangxun Lake (Figure 2). Isomer-specific biomagnification in aquatic food webs, leading to a highly linear PFOS isomer profile in fish, has previously been pointed out as a limitation of isomer profiles as a source tracking tool. ${ }^{42}$ However, it is noteworthy that serum samples of fishery employees had a significantly lower $(p=0.001)$ percentage of linear PFOS isomers (78\%) compared to the fish samples from Tangxun Lake ( $>90 \%)$, which was determined to be the primary exposure medium. Since all major branched PFOS isomers are more readily eliminated via urine ${ }^{29}$ compared to linear PFOS, a higher percentage of linear PFOS human serum compared to fish would be expected if direct exposure to PFOS could fully explain the concentrations in human serum of fishery employees. The discrepancy in PFOS isomer profiles between fishery employee serum and the primary exposure media may, therefore, indicate that FOSA and other PFOS precursors in fish, which were not quantified in this study, make an important contribution to the total human exposure.

PFAS Concentrations in Urine and Estimation of Renal Clearance Rates. The clearance rate of a chemical from the human body is an important parameter for estimating its hazard profile. Previous studies have investigated total elimination rates of PFHxS, PFOS, and PFOA in retired fluorochemical workers ${ }^{36}$ and renal clearance rates of longchain PFASs in background exposed individuals. ${ }^{29}$ However, there are limited data for elimination rates of short-chain PFASs, and it is currently not known how the exposure level may influence elimination kinetics. ${ }^{17,18,47,51,52}$ The concentrations of PFASs in urine samples are shown in Table 1. PFOS was the dominant homologue in urine samples of fishery employees and their family members with a median concentration of $4700 \mathrm{ng} / \mathrm{L}$ and $2440 \mathrm{ng} / \mathrm{L}$, respectively. PFBA, PFPeA, PFOA, PFBS, and PFHxS were detected in more than $80 \%$ of the samples, whereas a lower detection frequency was observed for PFHxA (41\%), PFHpA (74\%), PFNA (59\%), and PFDA (38\%). It is noteworthy that PFUnDA and PFDoDA were not detected in any urine samples, despite the highest concentrations of PFUnDA and PFDoDA in serum samples reaching $36.4 \mathrm{ng} / \mathrm{mL}$ and $20.6 \mathrm{ng} /$ $\mathrm{mL}$, respectively. Spearman rank correlation analysis showed that there were significant correlations between concentrations of PFASs in paired samples of serum and urine $(p<0.01)$, which was consistent with the results in the study by Zhang et $\mathrm{al}^{29}$ This indicates that the measurements of PFASs in urine can be used as markers of exposure to this group of contaminants.

On the basis of paired measurements of serum and urine, we calculated the daily renal clearance $\left(\mathrm{CL}_{\text {renal }} \mathrm{mL} / \mathrm{day} / \mathrm{kg}\right)$ of individual PFAS to assess the excretion efficiency of PFASs through urine (Table S8). Median renal clearance rates $\left(\mathrm{CL}_{\text {renal }}\right)$ of $\mathrm{C} 4$ to $\mathrm{C} 10$ perfluoroalkyl carboxylic acids (PFCAs) ranged from 0.020 to $16.5 \mathrm{~mL} / \mathrm{day} / \mathrm{kg}$. Long-chain PFCAs (C8-C10) had significantly lower clearance rates (0.020 to $0.069 \mathrm{~mL} / \mathrm{day} / \mathrm{kg}$ ) compared to short-chain PFCAs, and overall, there was a negative association between $\mathrm{CL}_{\text {renal }}$ and perfluoroalkyl chain length $(p=0.002)$. Perfluoroalkanesulfonic acids (PFSAs) displayed lower renal clearance rates ( 0.013 to $9.43 \mathrm{~mL} / \mathrm{day} / \mathrm{kg}$ ) compared to PFCAs of the same perfluoroalkyl chain-length, and a minimum in $\mathrm{CL}_{\text {renal }}$ was observed for perfluorohexane sulfonic acid $(\mathrm{PFHxS}) . \mathrm{CL}_{\text {renal }}$ values for PFOA, PFNA, PFDA, PFHxS, and PFOS from fishery employees were in general agreement with those previously reported in background exposed individuals from China and Japan. ${ }^{29,53}$ Furthermore, renal clearance rates were in agreement with total clearance rates determined for PFBS ${ }^{47}$ and PFOA, ${ }^{36}$ which provides confidence in the conclusion that PFCAs with less than eight perfluoroalkyl carbons are primarily eliminated via urine. ${ }^{29}$ However, further in line with the findings of Zhang et al., ${ }^{29}$ the renal clearance rates for PFHxS and PFOS were approximately 5 times slower than the total clearance rates estimated from retired fluorochemical workers. ${ }^{36}$ The discrepancy between renal clearance and total clearance rates for PFHxS and PFOS as determined by Olsen et al. provides further support to the conclusion that other routes of excretion are important to understanding total elimination. ${ }^{54}$ Thus, additional research to quantify fecal elimination of longchain PFCAs and PFSAs would help to understand the slow elimination of these compounds in humans and allow improved 
interpretation of biomonitoring data. Since renal excretion is a saturable process, ${ }^{51,52}$ elimination rates in humans may vary with the level of exposure. To examine if the renal clearance rates determined here were concentration dependent, the study participants were grouped with respect to exposure level (fishery employees, FM and BE). For PFOS, PFHxS, and PFOA, which displayed the largest range in serum concentrations, there were no statistically significant differences in renal clearance rates (Figure S3). The lack of a significant difference in $\mathrm{CL}_{\text {renal }}$ between fishery employees, family members, and the background-exposed reference group demonstrates that renal excretion rates of PFASs in humans may not be strongly concentration dependent at environmentally relevant exposure levels.

Short-chain PFCAs and PFBS showed renal clearance rates which were 2-3 orders of magnitude higher than those of the long-chain homologues. These data provide further support to the conclusion by others ${ }^{17,18,47}$ that short-chain PFASs have a relatively low accumulation potential in humans. Differences in renal clearance of PFCAs with differing chain lengths have primarily been attributed to their binding affinities to serum albumin and different sets of organic anion transporter proteins that govern the reabsorption of PFCAs from urine to blood. ${ }^{55-57}$ A recent study that investigated bovine serum albumin-water distribution coefficients $\left(K_{\mathrm{PW}}\right)$ demonstrated a positive association between binding affinities and the perfluoroalkyl chain, ${ }^{56}$ and the few data so far on human elimination of short-chain PFCAs indicate a substantially faster clearance of PFBA compared to PFHxA. ${ }^{17,18}$ Interestingly, our data displayed similar or slightly lower clearance rates for PFBA compared to PFHxA and PFHpA (Figure 3), although no significant differences were observed for clearance rates among these three PFCAs according to the statistical test. While the reasons for this observation remain unclear, it was proposed by Han et al. ${ }^{55}$ that the slower elimination of PFBA compared to PFHxA in rats may be due to a different set of transport proteins governing renal excretion. However, since PFHxA and PFHpA displayed a low detection frequency in both urine and serum, it should also be noted that estimated $\mathrm{CL}_{\text {renal }}$ values for these compounds are associated with a larger uncertainty compared to the other PFASs in this study. The large individual variability in clearance rates for PFBA and PFBS also indicate that further work is needed to better constrain these important parameters.

Risk Assessment and Blood Clinical Chemistry Detection. A preliminary risk assessment was performed to guide further epidemiological studies in the area. Calculated average daily intakes for fishery employees exposed from fish consumption were $1.48 \mathrm{ng} / \mathrm{kg}_{\mathrm{bw}} /$ day and $621 \mathrm{ng} / \mathrm{kg}_{\mathrm{bw}} /$ day for PFOA and PFOS, respectively (Table S7). Thus, estimated daily intake of PFOA was under the Tolerable Daily Intake (TDI) of 1500 for PFOA $\mathrm{ng} / \mathrm{kg}_{\mathrm{bw}} /$ day, whereas the mean PFOS intake by fishery employees and their families all significantly exceeded the TDI of 150 PFOS $\mathrm{ng} / \mathrm{kg}_{\mathrm{bw}} / \mathrm{day} .{ }^{46}$

Since previous studies have shown associations between PFASs and lipid, hepatic, and thyroid parameters in serum, ${ }^{23,24}$ CHO, LDL-chol, HDL-chol, TG, ALP, FT3, and FT4 were measured in this study. Multivariate regression model results of clinical chemistry on PFAS concentrations adjusted for age and BMI are given in Table S10. A statistically significant negative association was observed between PFHxS and TG (standardized coefficient $-1.67, p=0.02$ ), whereas a statistically significant positive association was observed between PFOS

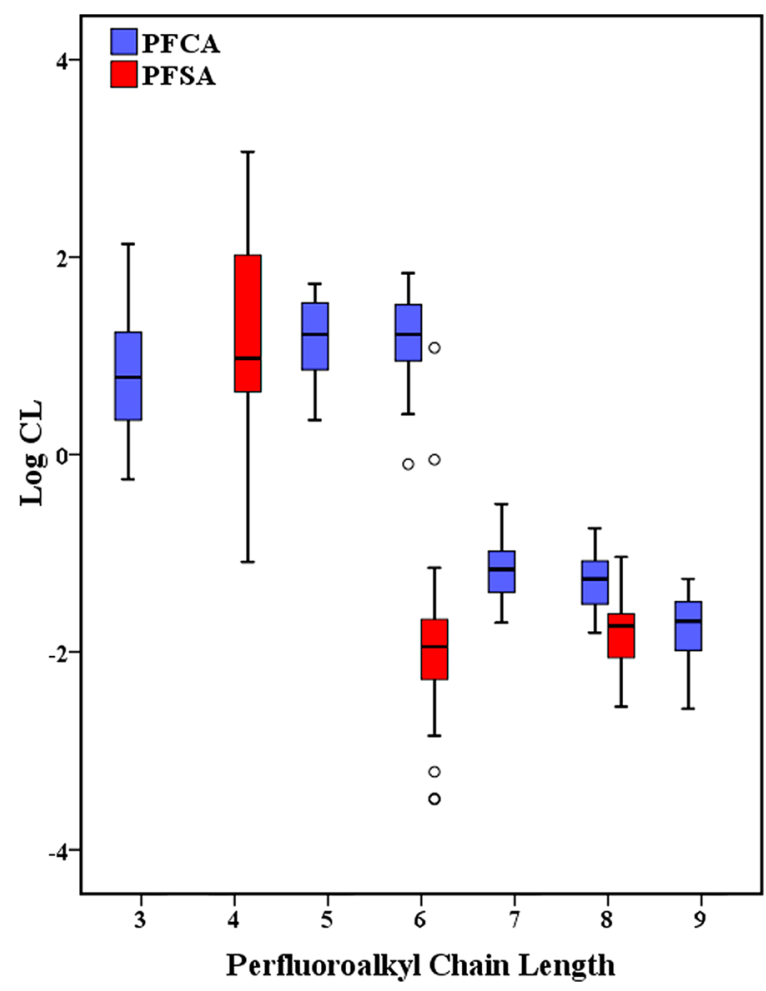

Figure 3. The relationship between the log $\mathrm{CL}$ (renal clearance, $\left.\mathrm{CL}_{\text {renal }}\right)$ and the perfluoroalkyl chain length $\left(\mathrm{C}\left(\mathrm{CF}_{2}\right)\right)$ of PFCAs and PFSAs. (The lower and upper limits of the whiskers indicate $5 \%$ and $95 \%$ values, respectively; boxes extend from 25 th to 75 th percentiles; horizontal lines within the boxes represent medians; circles below or above the whiskers indicate outlier values.)

and TG (standardized coefficient 1.79, $p=0.02$ ). However, there were no correlations between PFASs and other outcomes of serum lipid, such as HDL-chol, LDL-chol, and CHOL. Furthermore, the ratio of HDL-chol to LDL-chol, which has been considered a predictor of cardiovascular outcomes, ${ }^{58}$ showed no association with PFASs. The liver is an important target organ for PFAS toxicity, and liver enzymes were commonly measured for hepatic injury in clinical and epidemiologic studies. ${ }^{23,59}$ Similarly, in this study, a significantly positive correlation was found between PFOS and ALP (standardized coefficient 1.64, $p=0.03$ ). However, the results obtained so far do not indicate harmful effects of PFASs on the human liver (Table S9). Additionally, a significant negative association was observed between ALB and PFHxS (standardized coefficient $-1.53, p=0.02$ ), whereas a positive association was observed between ALB and PFOA (standardized coefficient $0.52, p=0.04$ ). In contrast to the study by Olsen and Zobel, ${ }^{24}$ there were no correlations between PFASs and serum thyroid hormones (FT3 and FT4) in our study.

Although certain outcomes of clinical chemistry detection were associated with PFASs, there were generally no indications of harmful effects to human health, as clinical parameters were mostly well within normal reference ranges (Table S9). It should, however, be pointed out that our study was limited by the relatively small population $(n=56)$ and potential selection biases for medical surveillance examinations. Previous studies have also reported inconsistent associations between clinical biomarkers, and PFAS exposure may to a large extent be explained by different study designs. ${ }^{25,60}$ More carefully designed prospective studies including a larger number of 
individuals should, therefore, be performed to evaluate the human health risks associated with PFAS exposure around Tangxun Lake.

Main Findings and Future Directions. The results presented in this paper provide unique data on external exposure media together with paired serum and urine samples from a human cohort with exceptionally high PFAS exposure. Key findings include the relationship between PFAS serum concentration and duration of exposure and estimations of renal clearance rates for a homologues series of PFASs. Also, the highly linear PFOS isomer profiles in fishery employees, reflecting the highly linear PFOS isomer profile in fish, is also interesting since it can help to constrain the applicability of PFOS isomer profiles as a biomarker of precursor exposure in other biomonitoring studies. ${ }^{42}$ Nevertheless, it should be mentioned that the analytical technique used in this study was not able to separate individual PFOS isomers as was done by, e.g., Beesoon et al. ${ }^{61}$ Thus, the quantification of branched PFOS isomers may be associated with a larger uncertainty compared to the previous study ${ }^{61}$ due to different response factors of individual isomers. ${ }^{62}$ Ongoing work in our laboratory will aim to address these issues by applying isomer-specific analytical methods to a range of PFASs. ${ }^{63}$

Estimated daily intakes demonstrate that fishery employees are at risk of health effects due to their high exposure to PFOS. However, the inconclusive results from correlations with blood serum parameters and PFOS highlight the need for further epidemiological studies in the Tangxun Lake area. We believe that the identification of a population around Tangxun Lake with PFAS serum concentrations ranging over 4 orders of magnitude and a clearly defined exposure source will probably help to resolve controversial epidemiological findings of PFASs. ${ }^{25,60}$

\section{ASSOCIATED CONTENT}

\section{S Supporting Information}

Additional information regarding chemicals and materials, sampling, sample pretreatment, quality assurance and quality control, blood clinical chemistry detection, data description and statistical analyses, Tables S1-S11, and Figures S1-S3. This material is available free of charge via the Internet at http:// pubs.acs.org/.

\section{AUTHOR INFORMATION}

\section{Corresponding Authors}

*Tel.: +86 (27) 84238886. E-mail: ly76@263.net.

*Tel.: +86 (10) 62849182. Fax: +86 (10) 62849182. E-mail: caiyaqi@rcees.ac.cn.

\section{Notes}

The authors declare no competing financial interest.

\section{ACKNOWLEDGMENTS}

This work was supported by National Key Basic Research Program of China (2014CB114402), National Natural Science Foundation of China (Nos. 41023005, 21377145, 21321004), State Environmental Protection Welfare Scientific Research Project (201009026), and the Program for New Century Excellent Talents in University (NCET-11-0964).

\section{REFERENCES}

(1) Kissa, E. Fluorinated Surfactants and Repellents. In Surfactant Science Series, CRC, 2nd ed.; Marcel Dekker: New York, 2001; Vol 97, pp 1-615.

(2) Hansen, K. J.; Clemen, L. A.; Ellefson, M. E.; Johnson, H. O. Compound-Specific, Quantitative Characterization of Organic Fluorochemicals in Biological Matrices. Environ. Sci. Technol. 2001, 35 (4), $766-770$.

(3) Houde, M.; Martin, J. W.; Letcher, R. J.; Solomon, K. R.; Muir, D. C. G. Biological Monitoring of Polyfluoroalkyl Substances: A Review. Environ. Sci. Technol. 2006, 40 (11), 3463-3473.

(4) Conder, J. M.; Hoke, R. A.; Wolf, W. d.; Russell, M. H.; Buck, R. C. Are PFCAs Bioaccumulative? A Critical Review and Comparison with Regulatory Criteria and Persistent Lipophilic Compounds. Environ. Sci. Technol. 2008, 42 (4), 995-1003.

(5) Vestergren, R.; Cousins, I. T. Tracking the Pathways of Human Exposure to Perfluorocarboxylates. Environ. Sci. Technol. 2009, 43 (15), 5565-5575.

(6) Andersen, M. E.; Butenhoff, J. L.; Chang, S.-C.; Farrar, D. G.; Kennedy, G. L.; Lau, C.; Olsen, G. W.; Seed, J.; Wallace, K. B. Perfluoroalkyl Acids and Related Chemistries-Toxicokinetics and Modes of Action. Toxicol. Sci. 2008, 102 (1), 3-14.

(7) $3 \mathrm{M}$ Company. Voluntary Use and Exposure Information Profile for Perfluorooctanoic Acid and Salts. USEPA Administrative Record AR226-0595. 2000a. Available from www.regulations.gov as document EPA-HQ-OPPT-2002-0051-0009.

(8) Listing of perfluorooctane sulfonic acid, its salts and perfluorooctane sulfonyl fluoride Stockholm Convention on Persistent Organic Pollutants (POPs). In Program, U. N. E., Ed. UNEP-POPSCOP. 4-SC-4-17: Geneva, Switzerland, 2009.

(9) Xie, S.; Wang, T.; Liu, S.; Jones, K. C.; Sweetman, A. J.; Lu, Y. Industrial source identification and emission estimation of perfluorooctane sulfonate in China. Environ. Int. 2013, 52 (0), 1-8.

(10) Buck, R. C.; Franklin, J.; Berger, U.; Conder, J. M.; Cousins, I. T.; de Voogt, P.; Jensen, A. A.; Kannan, K.; Mabury, S. A.; van Leeuwen, S. P. Perfluoroalkyl and polyfluoroalkyl substances in the environment: terminology, classification, and origins. Integr. Environ. Assess. Manage. 2011, 7 (4), 513-541.

(11) Haug, L. S.; Thomsen, C.; Brantsæter, A. L.; Kvalem, H. E.; Haugen, M.; Becher, G.; Alexander, J.; Meltzer, H. M.; Knutsen, H. K. Diet and particularly seafood are major sources of perfluorinated compounds in humans. Environ. Int. 2010, 36 (7), 772-778.

(12) Holzer, J.; Midasch, O.; Rauchfuss, K.; Kraft, M.; Reupert, R.; Angerer, J.; Kleeschulte, P.; Marschall, N.; Wilhelm, M. Biomonitoring of perfluorinated compounds in children and adults exposed to perfluorooctanoate-contaminated drinking water. Environ. Health Perspect. 2008, 116 (5), 651-657.

(13) Lorber, M.; Egeghy, P. P. Simple Intake and Pharmacokinetic Modeling to Characterize Exposure of Americans to Perfluoroctanoic Acid, PFOA. Environ. Sci. Technol. 2011, 45 (19), 8006-8014.

(14) Beesoon, S.; Genuis, S. J.; Benskin, J. P.; Martin, J. W. Exceptionally High Serum Concentrations of Perfluorohexanesulfonate in a Canadian Family are Linked to Home Carpet Treatment Applications. Environ. Sci. Technol. 2012, 46 (23), 12960-12967.

(15) Vestergren, R.; Berger, U.; Glynn, A.; Cousins, I. T. Dietary exposure to perfluoroalkyl acids for the Swedish population in 1999, 2005 and 2010. Environ. Int. 2012, 49 (0), 120-127.

(16) Glynn, A.; Berger, U.; Bignert, A.; Ullah, S.; Aune, M.; Lignell, S.; Darnerud, P. O. Perfluorinated Alkyl Acids in Blood Serum from Primiparous Women in Sweden: Serial Sampling during Pregnancy and Nursing, And Temporal Trends 1996-2010. Environ. Sci. Technol. 2012, 46 (16), 9071-9079.

(17) Russell, M. H.; Nilsson, H.; Buck, R. C. Elimination kinetics of perfluorohexanoic acid in humans and comparison with mouse, rat and monkey. Chemosphere 2013, 93 (10), 2419-2425.

(18) Chang, S. C.; Das, K.; Ehresman, D. J.; Ellefson, M. E.; Gorman, G. S.; Hart, J. A.; Noker, P. E.; Tan, Y. M.; Lieder, P. H.; Lau, C.; Olsen, G. W.; Butenhoff, J. L. Comparative pharmacokinetics of 
perfluorobutyrate in rats, mice, monkeys, and humans and relevance to human exposure via drinking water. Toxicol. Sci. 2008, 104 (1), 40-53. (19) Olsen, G. W.; Lange, C. C.; Ellefson, M. E.; Mair, D. C.; Church, T. R.; Goldberg, C. L.; Herron, R. M.; Medhdizadehkashi, Z.; Nobiletti, J. B.; Rios, J. A.; Reagen, W. K.; Zobel, L. R. Temporal Trends of Perfluoroalkyl Concentrations in American Red Cross Adult Blood Donors, 2000-2010. Environ. Sci. Technol. 2012, 46 (11), 6330-6338.

(20) Apelberg, B. J.; Witter, F. R.; Herbstman, J. B.; Calafat, A. M.; Halden, R. U.; Needham, L. L.; Goldman, L. R. Cord serum concentrations of perfluorooctane sulfonate (PFOS) and perfluorooctanoate (PFOA) in relation to weight and size at birth. Environ. Health Perspect. 2007, 115 (11), 1670-1676.

(21) Nelson, J. W.; Hatch, E. E.; Webster, T. F. Exposure to Polyfluoroalkyl Chemicals and Cholesterol, Body Weight, and Insulin Resistance in the General U.S. Population. Environ. Health Perspect. 2010, 118 (2), 197-202.

(22) Joensen, U. N.; Bossi, R.; Leffers, H.; Jensen, A. A.; Skakkebaek, N. E.; Jorgensen, N. Do Perfluoroalkyl Compounds Impair Human Semen Quality? Environ. Health Perspect. 2009, 117 (6), 923-927.

(23) Sakr, C. J.; Leonard, R. C.; Kreckmann, K. H.; Slade, M. D.; Cullen, M. R. Longitudinal Study of Serum Lipids and Liver Enzymes in Workers With Occupational Exposure to Ammonium Perfluorooctanoate. J. Occup. Environ. Med. 2007, 49 (8), 872-879.

(24) Olsen, G.; Zobel, L. Assessment of lipid, hepatic, and thyroid parameters with serum perfluorooctanoate (PFOA) concentrations in fluorochemical production workers. Int. Arch. Occup. Environ. Health 2007, 81 (2), 231-246.

(25) Steenland, K.; Fletcher, T.; Savitz, D. A. Epidemiologic evidence on the health effects of perfluorooctanoic acid (PFOA). Environ. Health Perspect. 2010, 118 (8), 1100-1108.

(26) Patel, C. J.; Cullen, M. R.; Ioannidis, J. P.; Butte, A. J. Systematic evaluation of environmental factors: persistent pollutants and nutrients correlated with serum lipid levels. Int. J. Epidemiol. 2012, 41 (3), 828843.

(27) Zhou, Z.; Liang, Y.; Shi, Y.; Xu, L.; Cai, Y. Occurrence and Transport of Perfluoroalkyl Acids (PFAAs), Including Short-Chain PFAAs in Tangxun Lake, China. Environ. Sci. Technol. 2013, 47 (16), 9249-9257.

(28) Yeung, L. W. Y.; Taniyasu, S.; Kannan, K.; Xu, D. Z. Y.; Guruge, K. S.; Lam, P. K. S.; Yamashita, N. An analytical method for the determination of perfluorinated compounds in whole blood using acetonitrile and solid phase extraction methods. J. Chromatogr. A 2009, 1216 (25), 4950-4956.

(29) Zhang, Y.; Beesoon, S.; Zhu, L.; Martin, J. W. Biomonitoring of Perfluoroalkyl Acids in Human Urine and Estimates of Biological HalfLife. Environ. Sci. Technol. 2013, 47 (18), 10619-10627.

(30) Borghi, L.; Meschi, T.; Amato, F.; Briganti, A.; Novarini, A.; Giannini, A. Urinary Volume, Water and Recurrences in Idiopathic Calcium Nephrolithiasis: A 5-year Randomized Prospective Study. J. Urol. (N. Y., NY, U. S.) 1996, 155 (3), 839-843.

(31) Zhang, T.; Sun, H. W.; Wu, Q.; Zhang, X. Z.; Yun, S. H.; Kannan, K. Perfluorochemicals in Meat, Eggs and Indoor Dust in China: Assessment of Sources and Pathways of Human Exposure to Perfluorochemicals. Environ. Sci. Technol. 2010, 44 (9), 3572-3579.

(32) Björklund, J. A.; Thuresson, K.; de Wit, C. A. Perfluoroalkyl Compounds (PFCs) in Indoor Dust: Concentrations, Human Exposure Estimates, and Sources. Environ. Sci. Technol. 2009, 43 (7), 2276-2281.

(33) Bao, J.; Liu, W.; Liu, L.; Jin, Y.; Dai, J.; Ran, X.; Zhang, Z.; Tsuda, S. Perfluorinated Compounds in the Environment and the Blood of Residents Living near Fluorochemical Plants in Fuxin, China. Environ. Sci. Technol. 2011, 45 (19), 8075-8080.

(34) Holzer, J.; Goen, T.; Just, P.; Reupert, R.; Rauchfuss, K.; Kraft, M.; Muller, J.; Wilhelm, M. Perfluorinated Compounds in Fish and Blood of Anglers at Lake Möhne, Sauerland Area, Germany. Environ. Sci. Technol. 2011, 45 (19), 8046-8052.

(35) Yeung, L. W. Y.; So, M. K.; Jiang, G.; Taniyasu, S.; Yamashita, N.; Song, M.; Wu, Y.; Li, J.; Giesy, J. P.; Guruge, K. S.; Lam, P. K. S.
Perfluorooctanesulfonate and Related Fluorochemicals in Human Blood Samples from China. Environ. Sci. Technol. 2006, 40 (3), 715720.

(36) Olsen, G. W.; Burris, J. M.; Ehresman, D. J.; Froehlich, J. W.; Seacat, A. M.; Butenhoff, J. L.; Zobel, L. R. Half-Life of Serum Elimination of Perfluorooctanesulfonate,Perfluorohexanesulfonate, and Perfluorooctanoate in Retired Fluorochemical Production Workers. Environ. Health. Perspect. 2007, 115 (9), 1298-1305.

(37) Nilsson, H.; Karrman, A.; Rotander, A.; van Bavel, B.; Lindstrom, G.; Westberg, H. Inhalation Exposure to Fluorotelomer Alcohols Yield Perfluorocarboxylates in Human Blood? Environ. Sci. Technol. 2010, 44 (19), 7717-7722.

(38) Olsen, G. W.; Logan, P. W.; Hansen, K. J.; Simpson, C. A.; Burris, J. M.; Burlew, M. M.; Vorarath, P. P.; Venkateswarlu, P.; Schumpert, J. C.; Mandel, J. H. An Occupational Exposure Assessment of a Perfluorooctanesulfonyl Fluoride Production Site: Biomonitoring. AIHA J. 2003, 64 (5), 651-659.

(39) Ye, X.; Strynar, M. J.; Nakayama, S. F.; Varns, J.; Helfant, L.; Lazorchak, J.; Lindstrom, A. B. Perfluorinated compounds in whole fish homogenates from the Ohio, Missouri, and Upper Mississippi Rivers, USA. Environ. Pollut. 2008, 156 (3), 1227-1232.

(40) Martin, J. W.; Whittle, D. M.; Muir, D. C. G.; Mabury, S. A. Perfluoroalkyl Contaminants in a Food Web from Lake Ontario. Environ. Sci. Technol. 2004, 38 (20), 5379-5385.

(41) Asher, B. J.; Wang, Y.; De Silva, A. O.; Backus, S.; Muir, D. C. G.; Wong, C. S.; Martin, J. W. Enantiospecific Perfluorooctane Sulfonate (PFOS) Analysis Reveals Evidence for the Source Contribution of PFOS-Precursors to the Lake Ontario Foodweb. Environ. Sci. Technol. 2012, 46 (14), 7653-7660.

(42) Martin, J. W.; Asher, B. J.; Beesoon, S.; Benskin, J. P.; Ross, M. S. PFOS or PreFOS? Are perfluorooctane sulfonate precursors (PreFOS) important determinants of human and environmental perfluorooctane sulfonate (PFOS) exposure? J. Environ. Monitor. 2010, 12 (11), 1979-2004.

(43) Benskin, J. P.; Ikonomou, M. G.; Gobas, F. A. P. C.; Begley, T. H.; Woudneh, M. B.; Cosgrove, J. R. Biodegradation of N-Ethyl Perfluorooctane Sulfonamido Ethanol (EtFOSE) and EtFOSE-Based Phosphate Diester (SAmPAP Diester) in Marine Sediments. Environ. Sci. Technol. 2013, 47 (3), 1381-1389.

(44) Tomy, G. T.; Tittlemier, S. A.; Palace, V. P.; Budakowski, W. R.; Braekevelt, E.; Brinkworth, L.; Friesen, K. Biotransformation of NEthyl Perfluorooctanesulfonamide by Rainbow Trout (Onchorhynchus mykiss) Liver Microsomes. Environ. Sci. Technol. 2004, 38 (3), $758-762$.

(45) Mak, Y. L.; Taniyasu, S.; Yeung, L. W. Y.; Lu, G.; Jin, L.; Yang, Y.; Lam, P. K. S.; Kannan, K.; Yamashita, N. Perfluorinated Compounds in Tap Water from China and Several Other Countries. Environ. Sci. Technol. 2009, 43 (13), 4824-4829.

(46) EFSA. Perfluorooctane sulfonate (PFOS), perfluorooctanoic acid (PFOA) and their salts. Scientific opinion of the panel on contamininants in the food chain, 2008, available at http://www.efsa. europa.eu/EFSA/efsa_locale-1178620753812_1211902012410.htm.

(47) Olsen, G. W.; Chang, S.-C.; Noker, P. E.; Gorman, G. S.; Ehresman, D. J.; Lieder, P. H.; Butenhoff, J. L. A comparison of the pharmacokinetics of perfluorobutanesulfonate (PFBS) in rats, monkeys, and humans. Toxicology 2009, 256 (1-2), 65-74.

(48) Ross, M. S.; Wong, C. S.; Martin, J. W. Isomer-Specific Biotransformation of Perfluorooctane Sulfonamide in SpragueDawley Rats. Environ. Sci. Technol. 2012, 46 (6), 3196-3203.

(49) Benskin, J. P.; Holt, A.; Martin, J. W. Isomer-Specific Biotransformation Rates of a Perfluorooctane Sulfonate (PFOS)Precursor by Cytochrome P450 Isozymes and Human Liver Microsomes. Environ. Sci. Technol. 2009, 43 (22), 8566-8572.

(50) Benskin, J. P.; De Silva, A. O.; Martin, L. J.; Arsenault, G.; Mccrindle, R.; Riddell, N.; Mabury, S. A.; Martin, J. W. Disposition of Perfluorinated Acid Isomers in Sprague-Dawley Rats; Part 1: Single Dose. Environ. Toxicol. Chem. 2009, 28 (3), 542-554.

(51) Loccisano, A. E.; Campbell, J. L., Jr; Andersen, M. E.; Clewell Iii, H. J. Evaluation and prediction of pharmacokinetics of PFOA and 
PFOS in the monkey and human using a PBPK model. Regul. Toxicol. Pharmacol. 2011, 59 (1), 157-175.

(52) Andersen, M. E.; Clewell, H. J., III; Tan, Y.-M.; Butenhoff, J. L.; Olsen, G. W. Pharmacokinetic modeling of saturable, renal resorption of perfluoroalkylacids in monkeys-Probing the determinants of long plasma half-lives. Toxicology 2006, 227 (1-2), 156-164.

(53) Harada, K.; Inoue, K.; Morikawa, A.; Yoshinaga, T.; Saito, N.; Koizumi, A. Renal clearance of perfluorooctane sulfonate and perfluorooctanoate in humans and their species-specific excretion. Environ. Res. 2005, 99 (2), 253-261.

(54) Harada, K. H.; Hashida, S.; Kaneko, T.; Takenaka, K.; Minata, M.; Inoue, K.; Saito, N.; Koizumi, A. Biliary excretion and cerebrospinal fluid partition of perfluorooctanoate and perfluorooctane sulfonate in humans. Environ. Toxicol. Pharmacol. 2007, 24 (2), 134139.

(55) Han, X.; Nabb, D. L.; Russell, M. H.; Kennedy, G. L.; Rickard, R. W. Renal Elimination of Perfluorocarboxylates (PFCAs). Chem. Res. Toxicol. 2012, 25 (1), 35-46.

(56) Bischel, H. N.; MacManus-Spencer, L. A.; Zhang, C.; Luthy, R. G. Strong associations of short-chain perfluoroalkyl acids with serum albumin and investigation of binding mechanisms. Environ. Toxicol. Chem. 2011, 30 (11), 2423-2430.

(57) Weaver, Y. M.; Ehresman, D. J.; Butenhoff, J. L.; Hagenbuch, B. Roles of Rat Renal Organic Anion Transporters in Transporting Perfluorinated Carboxylates with Different Chain Lengths. Toxicol. Sci. 2010, 113 (2), 305-314.

(58) Fernandez, M. L.; Webb, D. The LDL to HDL Cholesterol Ratio as a Valuable Tool to Evaluate Coronary Heart Disease Risk. J. Am. Coll. Nutr. 2008, 27 (1), 1-5.

(59) Lin, C.-Y.; Lin, L.-Y.; Chiang, C.-K.; Wang, W.-J.; Su, Y.-N.; Hung, K.-Y.; Chen, P.-C. Investigation of the Associations Between Low-Dose Serum Perfluorinated Chemicals and Liver Enzymes in US Adults. Am. J. Gastroenterol. 2009, 105, 1354-1363.

(60) Fletcher, T.; Galloway, T. S.; Melzer, D.; Holcroft, P.; Cipelli, R.; Pilling, L. C.; Mondal, D.; Luster, M.; Harries, L. W. Associations between PFOA, PFOS and changes in the expression of genes involved in cholesterol metabolism in humans. Environ. Int. 2013, 57$58(0), 2-10$.

(61) Beesoon, S.; Webster, G. M.; Shoeib, M.; Harner, T.; Benskin, J. P.; Martin, J. W. Isomer Profiles of Perfluorochemicals in Matched Maternal, Cord, and House Dust Samples: Manufacturing Sources and Transplacental Transfer. Environ. Health. Perspect. 2011, 119 (11), 1659-1664.

(62) Berger, U.; Kaiser, M.; Kärrman, A.; Barber, J.; Leeuwen, S. J. Recent developments in trace analysis of poly- and perfluoroalkyl substances. Anal. Bioanal. Chem. 2011, 400 (6), 1625-1635.

(63) Benskin, J. P.; Bataineh, M.; Martin, J. W. Simultaneous Characterization of Perfluoroalkyl Carboxylate, Sulfonate, and Sulfonamide Isomers by Liquid Chromatography-Tandem Mass Spectrometry. Anal. Chem. 2007, 79 (17), 6455-6464. 